\title{
Neoadjuvant vs. adjuvant chemotherapy in locally advanced non- small cell lung cancer-is timing everything?
}

\author{
Melanie P. Subramanian, Varun Puri \\ Division of Cardiothoracic Surgery, Washington University School of Medicine, St. Louis, MO, USA \\ Correspondence to: Melanie P. Subramanian, MD, MPHS. Division of Cardiothoracic Surgery, Washington University School of Medicine, S. Euclid \\ Avenue, Campus Box 8234, St. Louis, MO 63110, USA. Email: m.p.subramanian@email.wustl.edu. \\ Provenance: This is an invited article commissioned by the Academic Editor Dr. Shuangjiang Li (Department of Thoracic Surgery and West China \\ Medical Center, West China Hospital, Sichuan University, Chengdu, China). \\ Comment on: Brandt WS, Yan W, Zhou J, et al. Outcomes after neoadjuvant or adjuvant chemotherapy for cT2-4N0-1 non-small cell lung cancer: A \\ propensity-matched analysis. J Thorac Cardiovasc Surg 2019;157:743-753.e3.
}

Submitted Nov 22, 2019. Accepted for publication Dec 02, 2019.

doi: $10.21037 /$ jtd.2019.12.40

View this article at: http://dx.doi.org/10.21037/jtd.2019.12.40

Current standard of care for the multimodal management of early, locally advanced non-small cell lung cancer (NSCLC) recommends surgical resection followed by adjuvant therapy (1). These guidelines have been informed by an abundance of level one evidence comparing surgical resection with adjuvant therapy to surgical resection alone $(2,3)$. However, the role and potential benefits of neoadjuvant therapy in the same population are less understood.

Brandt and colleagues attempted to shed light on the debate between neoadjuvant and adjuvant therapy for stage IB-III, N0-1 disease (4). They performed a propensitymatched retrospective study comparing neoadjuvant to adjuvant therapy regimens in patients with cT2-4N0-1M0 NSCLC. Using longitudinal data collected from a single tertiary care center, the authors attempted to compare their primary outcome of disease-free survival (DFS) between treatment cohorts. Their secondary outcomes included overall survival (OS) and chemotherapy regimen compliance. They analyzed data from 92 matched pairs and ultimately demonstrated that there was no significant difference in DFS and OS between treatment cohorts.

The findings from Brandt et al.'s study are similar to previously published studies in the limited pool of literature that performed direct comparisons between neoadjuvant and adjuvant therapy. The NATCH trial represents the only published phase III trial that allows for direct comparison between neoadjuvant and adjuvant chemotherapy (5).
This trial recruited 624 patients with stage I-IIIA, N0-N1 NSCLC and randomized them to three treatment arms: surgery followed by adjuvant chemotherapy, neoadjuvant chemotherapy followed by surgery, and surgery alone. Patients assigned to receive chemotherapy received three cycles of paclitaxel-carboplatin. The primary endpoint for this study was DFS. The study ultimately found there was no difference in DFS between treatment arms. Lim and colleagues published a meta-analysis of trials that have compared either postoperative or preoperative chemotherapy to surgery-alone in an attempt to extract a head-to-head comparison between preoperative and postoperative chemotherapy strategies (6). They abstracted data from 32 trials that enrolled over 10,000 patients, with 22 trials studying postoperative chemotherapy and the remainder studying preoperative chemotherapy. Their analysis failed to find any difference in DFS or OS between treatment strategies.

While the majority of the available literature does not support the use of neoadjuvant chemotherapy compared to adjuvant chemotherapy, there are some theoretical benefits that have not been specifically addressed nor adequately studied. Administration of neoadjuvant chemotherapy could have the potential to preoperatively reduce tumor size, eradicate micro-metastases, and provide important prognostic information based on radiographic response (7). Brandt and colleagues demonstrated this in their subgroup analysis evaluating radiographic changes in disease burden 
using Response Evaluation Criteria in Solid Tumors (RECIST) (4). They showed that degree of radiographic response based on RECIST criteria was significantly associated with DFS, with $15 \%$ and $64 \%$ of the neoadjuvant chemotherapy cohort exhibiting a major pathologic and partial response, respectively.

While the majority of studies have focused on traditional endpoints of DFS and OS, there are additional outcomes that are important when considering the entire clinical context of a patient's treatment course. One of the major drawbacks of adjuvant therapy is that patients may not initiate or complete treatment due to postoperative complications or diminished tolerance. Thus, providing chemotherapy upfront has the potential to offer greater completion rates, especially in patients where frailty is a concern. Brandt and colleagues measured compliance to chemotherapy regimens and demonstrated that patients who received neoadjuvant chemotherapy compared to adjuvant chemotherapy were more likely to receive the full dose strength (78\% vs. $63 \%$ ) and full cycle regimen $(91 \%$ vs. $78 \%$ ) (4). Additionally, patients who received adjuvant chemotherapy were more than twice as likely to experience an adverse reaction compared to patients who underwent neoadjuvant treatment ( $38 \%$ vs. $15 \%$ ). These findings were similar to those of the NATCH trial, where $97 \%$ of neoadjuvant chemotherapy patients initiated their planned course of treatment compared to only $66.2 \%$ of adjuvant therapy patients (5).

Perhaps the question that needs to be asked going forward is not if neoadjuvant therapy is better, but for whom is it better? Previous studies (including Brandt et al.'s) have attempted to identify similar patient cohorts, either through randomized selection or propensity score matching, to perform comparative analysis. However, there may be a distinct population of patients that may benefit from neoadjuvant chemotherapy based on individual patient or disease-related factors. Maclean and colleagues queried the National Cancer Database to compare neoadjuvant and adjuvant chemotherapy in patients with stage II and III NSCLC (8). Additionally, they identified characteristics associated with receipt of neoadjuvant chemotherapy. Their analysis, which included 35,134 patients, found that receipt of neoadjuvant chemotherapy had improved survival outcomes compared to surgery alone. They identified African American race, Charlson-Deyo Comorbidity score $>0$, treatment at a non-academic center, lower income status, and public insurance/no insurance were factors associated with less likelihood of receiving neoadjuvant chemotherapy. However, important patient and disease characteristics including patient frailty, tumor size, tumor histology, and tumor location were not assessed.

Brandt and colleagues should be applauded for their study and their contribution to the relatively limited pool of literature on this subject. In comparison to previous studies, they have included a lower percentage of stage IB patients, who are less likely to benefit from addition of chemotherapy $(2,4)$. Additionally, they used a small but clinically enriched dataset that includes patient and diseaserelated characteristics and endpoints that are not captured in most large clinical databases. However, the majority of studies have previously attempted to identify patients in which there is equipoise in offering neoadjuvant or adjuvant therapy. However, it is possible that there is not true equipoise for some patients, and it would be helpful to understand why certain patients were offered neoadjuvant therapy as opposed to adjuvant therapy. Future studies will be necessary to further our understanding of the role of neoadjuvant therapy in locally advanced, N0-N1 NSCLC.

\section{Acknowledgments}

None.

\section{Footnote}

Conflicts of Interest: The authors have no conflicts of interest to declare.

Ethical Statement: The authors are accountable for all aspects of the work in ensuring that questions related to the accuracy or integrity of any part of the work are appropriately investigated and resolved.

\section{References}

1. Ettinger DS, Aisner DL, Wood DE, et al. NCCN Guidelines Insights: Non-Small Cell Lung Cancer, Version 5.2018. J Natl Compr Canc Netw 2018;16:807-21.

2. McElnay P, Lim E. Adjuvant or neoadjuvant chemotherapy for NSCLC. J Thorac Dis 2014;6 Suppl 2:S224-7.

3. Pignon JP, Tribodet H, Scagliotti GV, et al. Lung adjuvant cisplatin evaluation: a pooled analysis by the LACE Collaborative Group. J Clin Oncol 2008;26:3552-9.

4. Brandt WS, Yan W, Zhou J, et al. Outcomes after neoadjuvant or adjuvant chemotherapy for cT2-4N0-1 non-small cell lung cancer: A propensity-matched analysis. 
J Thorac Cardiovasc Surg 2019;157:743-753.e3.

5. Felip E, Rosell R, Maestre JA, et al. Preoperative chemotherapy plus surgery versus surgery plus adjuvant chemotherapy versus surgery alone in early-stage nonsmall-cell lung cancer. J Clin Oncol 2010;28:3138-45.

6. Lim E, Harris G, Patel A, et al. Preoperative versus postoperative chemotherapy in patients with resectable non-small cell lung cancer: systematic review and indirect comparison meta-analysis of randomized trials. J Thorac

Cite this article as: Subramanian MP, Puri V. Neoadjuvant $v s$. adjuvant chemotherapy in locally advanced non-small cell lung cancer—is timing everything? J Thorac Dis 2019;11(12):56745676. doi: $10.21037 /$ jtd.2019.12.40
Oncol 2009;4:1380-8.

7. NSCLC Meta-analysis Collaborative Group. Preoperative chemotherapy for non-small-cell lung cancer: a systematic review and meta-analysis of individual participant data. Lancet 2014;383:1561-71.

8. MacLean M, Luo X, Wang S, et al. Outcomes of neoadjuvant and adjuvant chemotherapy in stage 2 and 3 non-small cell lung cancer: an analysis of the National Cancer Database. Oncotarget 2018;9:24470-9. 